\title{
The "Cutaneous Rabbit" Hopping out of the Body
}

\author{
Makoto Miyazaki, ${ }^{1,2}$ Masaya Hirashima, ${ }^{3}$ and Daichi Nozaki ${ }^{3,4}$ \\ ${ }^{1}$ Research Institute of Kochi University of Technology, Tosayamada-cho, Kochi 782-8502, Japan, ${ }^{2}$ Future Institute for Sport Sciences, Waseda University, \\ Shinjuku-ku, Tokyo 162-0041, Japan, ${ }^{3}$ Graduate School of Education, The University of Tokyo, Bunkyo-ku, Tokyo 113-0033, Japan, and ${ }^{4}$ National Institute \\ of Information and Communications Technology, Keihanna Science City, Kyoto 619-0288, Japan
}

Rapid sequential taps delivered first to one location and then to another on the skin create the somatosensory illusion that the tapping is occurring at intermediate locations between the actual stimulus sites, as if a small rabbit were hopping along the skin from the first site to the second (called the "cutaneous rabbit"). Previous behavioral studies have attributed this illusion to the early unimodal somatosensory body map. A functional magnetic resonance imaging study recently confirmed the association of the illusion with somatotopic activity in the primary somatosensory cortex. Thus, the cutaneous rabbit illusion has been confined to one's own body. In the present paper, however, we show that the cutaneous rabbit can "hop out of the body" onto an external object held by the subject. We delivered rapid sequential taps to the left and right index fingers. When the subjects held a stick such that it was laid across the tips of their index fingers and received the taps via the stick, they reported sensing the illusory taps in the space between the actual stimulus locations (i.e., along the stick). This suggests that the cutaneous rabbit effect involves not only the intrinsic somatotopic representation but also the representation of the extended body schema that results from body-object interactions.

\section{Introduction}

The brain possesses a representation of the body map in the primary somatosensory cortex (S1) (Penfield and Boldrey, 1937). The somatotopic map reflects the locations of physical stimuli on the skin. Our somatosensory perception, however, does not always correspond to the actual locations being stimulated; rather, it is often accompanied by illusionary sensations. One well known example is the "cutaneous rabbit" (Geldard and Sherrick, 1972). When a series of rapid taps are delivered first at one location on the skin and then at another, without a break in regularity, the recipient perceives illusory taps between the actual stimulation locations as if a small rabbit were hopping along the skin from the first site to the second. The illusion has attracted interest not only from neuroscientists and psychologists but also from philosophers (Dennett, 1991), because it demonstrates the relativity or interdependency of space and time in somatesthesia (Helson, 1930; Jones, 1956; Goldreich, 2007) and, furthermore, that sensory events at a certain time point are influenced by future sensory events. This seemingly mysterious, retroactive phenomenon indicates that our conscious perception does not merely reflect crude sensory inputs but is created after integrating sensory inputs that occur within a certain time window. Such an effect has been termed "postdiction" (Eagleman and Sejnowski, 2000), and it is now accepted as one of the key concepts that characterize our conscious perception.

Received Aug. 9, 2009; accepted Dec. 16, 2009.

This research was partially supported by the Ministry of Education, Science, Sports, and Culture, Grants-in-Aid for Young Scientists (S) 21670004 (M.M.), (S) 20670008 (D.N.), and (Start-up) 21800015 (M.H.). We thank S. Kitazawa,

S. Yamamoto, and Y. Sato for helpful comments on this study.

This article is freely available online through the J Neurosci Open Choice option.

Correspondence should be addressed to Makoto Miyazaki, Research Institute of Kochi University of Technology,

185 Miyanokuchi, Tosayamada-cho, Kochi 782-8502, Japan. E-mail: miyazaki.makoto@kochi-tech.ac.jp.

DOI:10.1523/JNEUROSCI.3887-09.2010

Copyright $\odot 2010$ the authors $\quad 0270-6474 / 10 / 301856-05 \$ 15.00 / 0$
For years, researchers have attempted to determine the mechanisms responsible for the cutaneous rabbit illusion. Geldard and Sherrick measured the degree of the sensation at various body sites and reported that the amplitude of the sensory saltation was in accordance with the size of the somatosensory receptive field constructed in S1 (Geldard, 1982; Geldard and Sherrick, 1983). Additionally, the illusory rabbit did not cross the body midline (Geldard, 1982), which is consistent with the basic definition that tactile information is represented in a lateralized manner up to $S 1$ (Mountcastle, 1984). It was therefore argued that the cutaneous rabbit illusion is attributable to early somatotopic activity in S1. This argument is supported by a recent study using behavioral measurements and theoretical models (Flach and Haggard, 2006), but it has been challenged by reports that higher factors [e.g., selective attention (Kilgard and Merzenich, 1995) and across-arm effects (Eimer et al., 2005)] influence the illusory effect. The use of the latest brain-imaging techniques, however, has settled the debate. A recent functional magnetic resonance imaging (fMRI) study showed that, when subjects experienced the cutaneous rabbit illusion, there was a correlated activation in the somatotopic region in S1 [Brodmann area (BA) 1] that corresponded to the skin site where the illusory sensation occurred (Blankenburg et al., 2006). Thus, it was confirmed that the cutaneous rabbit illusion is associated with the early sensory body map representation in S1. Here, we show that the cutaneous rabbit sensation, which should be a somatotopic effect, can be experienced as leaving the body and hopping onto a stick held by both index fingers. This effect occurs although the stick, by definition, lacks a specific receptive field in S1.

\section{Materials and Methods}

Stimulation with a stick (experiment 1)

Subjects. Eight subjects (seven male, one female; seven right handed, one left handed; $19-25$ years of age) participated. All subjects were naive to 


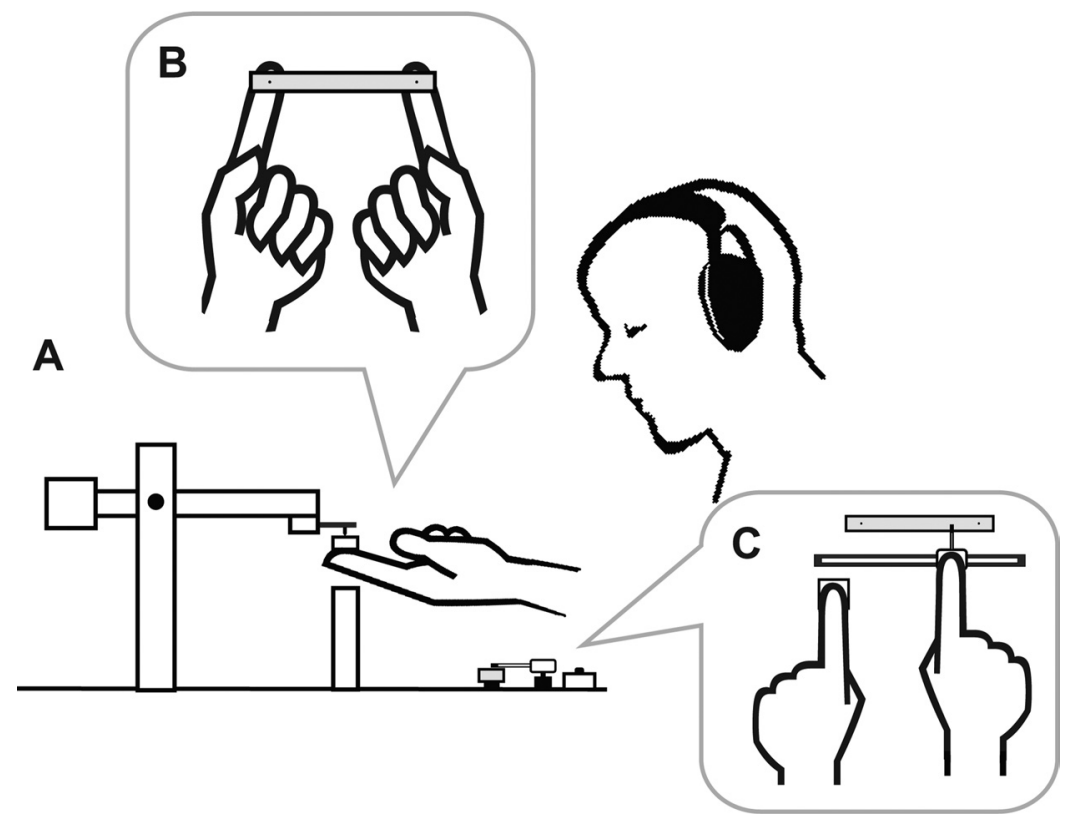

Figure 1. Overview of the experimental setup. $A$, Side view of a subjectholding the piezoelectric contactors with a flat stick. $B$, Bird's eye view of a subject's fingers holding the stick. $C$, Bird's eye view of the pointer set that was used for reporting the perceptual experiences.

the purpose of the study. Written informed consent was obtained from each individual before the experiments were performed. This study was approved by the institutional ethics committee.

Apparatuses and stimuli. Subjects received mechanical pulses at the ventral pads of the right and left index fingers via a flat stick (Fig. $1 A, B$ ). The stick and contactors were supported by the subjects without any additional support. The subjects opened their eyes when they lifted up the contactors and the stick in the beginning of each trial. During the pulse delivery, however, the subjects closed their eyes.

The mechanical pulses were generated by two sets of piezoelectric contactor systems (custom-made; Uchida Dennsi). Each contactor was attached to the tip of a balanced pendulum (Fig. $1 \mathrm{~A}$ ), and the static weight was set at $11 \mathrm{~g}$. The distance between the contactors was $80 \mathrm{~mm}$. The head of the contactor was made of acrylonitrile butadiene styrene resin. It was $2 \mathrm{~mm}$ in diameter and $3.8 \mathrm{~mm}$ in height, and its pinpoint was gently sharpened. The contactors produced a small movement such that they pressed down the stick. The pulse reached its peak amplitude after 2 $\mathrm{ms}$ and returned to the initial position $2 \mathrm{~ms}$ later. The peak pulse amplitude was $0.08 \mathrm{~mm}$ under the condition that the contactor was not in contact with any object. This stimulus amplitude was commonly used in the experimental sessions for all subjects; the amplitude, on average, corresponded to $\sim 10$ times that of the sensory threshold (for details, see supplemental Table S1, available at www.jneurosci.org as supplemental material).

The stick used in experiment 1 was made of aluminum and measured $100 \times 10 \times 5 \mathrm{~mm}$. Two black dots $(1.5 \mathrm{~mm}$ in diameter $)$ were printed on the upper surface of the stick, one $10 \mathrm{~mm}$ from each end; the distance between the dots was $80 \mathrm{~mm}$. When subjects held the stick, the centers of the finger pads were placed at the points under the dots, and the heads of the contactors were put on the dots.

The pulse sequence given to subjects was composed of three pulses (Fig. 2A). The first pulse (P1) was delivered to the first location (L1). The second pulse (P2) was delivered to L1 $800 \mathrm{~ms}$ after P1, and, 50 or $80 \mathrm{~ms}$ after P2, the third pulse (P3) was delivered to the second location (L2). For half of the trials, L1 was on the right index finger and L2 was on the left index finger; the locations were reversed for the other half. The threetap sequence was optimal for generating the "reduced" rabbit (Flach and Haggard, 2006), which is a widely used simple version of the cutaneous rabbit illusion (Geldard, 1982; Geldard and Sherrick, 1983; Kilgard and Merzenich, 1995). In this sequence, P1 was temporally separated from P2 and P3 such that the sensation from P1 should not be affected by the latter two stimuli. In this case, P1 served as the "reference" that was free from the postdictive effect. In contrast, P2 was called the "attractee" and P3 was the "attractor (or attractant)." Previous studies observed that $\mathrm{P} 2$ was perceptually displaced toward $\mathrm{P} 3$ on the skin.

After the pulse delivery, subjects reported their perceptual experiences using a pointer set (Fig. 1C) in which a slide-type pointer device moved along another stick identical to that used during the mechanical stimulation. Subjects moved the pointer with their right hand to positions corresponding to where they experienced tapping sensations. To mark each location, they clicked a button with their left hand.

During the task, subjects were seated and wore headphones and sound-isolating earphones. The headphones played white noise and the earphones emitted beeps. Thus, the subjects could not hear the sound of the mechanical pulses; they could hear only the beeps and feel the tactile stimuli. The beeps instructed subjects to lift up the contactors and the stick in the beginning of each trial and to close their eyes before the pulse delivery.

Subjects were instructed not to limit their tactile attention to a specific point but to pay their tactile attention to the whole of the stick. Although they were informed of the number of taps (i.e., three) during the explanation of the task, they were required to report their sensation with the tap number just as they felt them when they felt fewer or greater than three taps.

In the experimental sessions, subjects reported only three taps. Although one subject reported four taps in one trial in experiments 1, it was less than in experiment 2 (see below, Data analysis). Additionally, in the interview immediately after the tasks, all subjects answered without hesitation that they never felt any tapping sensation, including faint sensations, other than those they reported using the pointer set. Subjects thus perceived just one tap at one point corresponding to one pulse. Moreover, the subjects reported the first pulse (P1), which was free from the effect of the other stimuli, only around the stimulated point L1 (Fig. 2). Thus, the single pulse via the stick gave subjects only one tapping sensation on the one finger that was placed under the contactor generating the pulse.

Procedure and conditions. In response to a short $(200 \mathrm{~ms})$ beep at the beginning of each trial, subjects put both of their index fingers on the lower surface of the stick so that the centers of the finger pads were placed under the right- and left-side dots printed on the upper surface of the stick, and they lifted up the stick from a pedestal. The subjects then placed the dots at the tips of the contactor heads and lifted up the two contactors with the stick. They were instructed to keep the contactors at a height where the arms of the contactors were approximately horizontal. At 8000 $\mathrm{ms}$ after the initial beep, a long (2000 ms) beep signaled the subjects to close their eyes. At $500 \mathrm{~ms}$ after the end of the long beep, the pulse sequence was delivered. After completion of the pulse delivery, the subjects opened their eyes and returned the stick to the pedestal. Using the pointer set, they then reported the locations where tapping sensations occurred in the order that they felt them. The intertrial interval (interval between the beginning of each trial) was $25 \mathrm{~s}$.

Subjects performed six blocks ( 12 trials each) of the task. In each block, L1 was allocated to the right index finger and L2 to the left index finger for half of the trials, and the locations were reversed for the other half. The interstimulus interval (ISI) between P2 and P3 was $50 \mathrm{~ms}$ for half of the trials and $80 \mathrm{~ms}$ for the other half. Each block therefore contained four types of sequences [two P1 allocations (left or right) $\times$ two ISI conditions (50 or $80 \mathrm{~ms}$ )], which were presented to subjects at random. Before the experimental session, the subjects performed 4-12 practice trials (one to three trials for each type of stimulus sequence) to familiarize themselves with the task. The number of practice trials depended on each subject's understanding of the task procedure and stability of responses. After the 
experimental session, the sensory threshold was measured (for details, see supplemental Table S1, available at www.jneurosci.org as supplemental material).

Stimulation without a stick (experiment 2) Eight subjects (six male, two female; all right handed; 19-23 years of age) participated in this experiment. Four of the subjects had participated in experiment 1 , and the other four were newly recruited. In this experiment, the subjects held a pair of square plates instead of a stick on the ventral pads of both index fingers (Fig. $3 B$ ). The plates were made of aluminum and measured $10 \times 10 \times 5 \mathrm{~mm}$. A black dot ( $1.5 \mathrm{~mm}$ in diameter) was printed at the center on the upper surface of each plate. Another pair of plates was installed in a pointer set to report the tapped positions. The other settings and procedures were the same as in experiment 1 . The sensory threshold of the subjects in experiment 2 was not different from that in experiment 1 (for details, see supplemental Table S1, available at www.jneurosci.org as supplemental material), indicating that there was no difference in the basic stimulus intensity or the sensory ability of the subjects between the two experiments.

\section{Data analysis}

Subjects reported three taps in almost all trials. The first, second, and third taps reported by the subjects should have reflected their sensations of P1, P2, and P3, respectively. The three taps were ordinarily reported in the spatial order from L1 to L2. For example, in experiment 1, $\mathrm{P} 1$ was reported at $\mathrm{L} 1, \mathrm{P} 2$ was displaced toward L2, and P3 was farther toward L2 (Fig. $2 B$ ). We measured the deviations of the reported taps from the actual stimulus points to estimate the illusory effect.

We removed the following exceptional reports from the data. Although none of the subjects reported two taps, four subjects reported four taps in some of the trials (one trial in one subject in experiment 1 ; one trial in two subjects, and three trials in one subject in experiment 2). In addition, subjects occasionally reported P2 closer to L2 than P3 [6.3 \pm $3.0 \%$ (mean \pm SEM) of trials for the $50 \mathrm{~ms}$ ISI and $4.9 \pm 3.0 \%$ for the 80 ms ISI in experiment $1 ; 5.6 \pm 4.8 \%$ for the $50 \mathrm{~ms}$ ISI and $4.5 \pm 3.9 \%$ for the $80 \mathrm{~ms}$ ISI in experiment 2]. This could be associated with the reversal of subjective temporal order that was stochastically observed in temporal order judgment (TOJ) of the short-interval stimuli. In previous papers, the stimulus intervals that yielded $84 \%$ correct judgments were $60-70 \mathrm{~ms}$ in TOJ of two stimuli delivered one to each hand without arm crossing (Yamamoto and Kitazawa, 2001; Miyazaki et al., 2006). According to this, the probability of the reversal of subjective order was predicted to be $\sim 20 \%$ in the $50 \mathrm{~ms}$ ISI case and $10 \%$ in the $80 \mathrm{~ms}$ ISI case, which were greater than the reversal probabilities in our data. It seems that the lower reversal probability in our task was attributable to the existence of the reference signal (P1). We speculate that P1 also played the role of an indicator of the order and direction of the two subsequent stimuli.

\section{Results}

In experiment 1 , the cutaneous rabbit illusion was observed. Figure $2 B$ shows a typical report from the subjects. The reference stimulus P1 (blue dot) was reported at the actual stimulus location L1. Conversely, the report of P2 (green dot) was displaced from L1 toward L2, and that of P3 (orange dot) was displaced from its actual stimulus location L2 to L1. In addition, the subjects often reported the perceptual displacement of P2 but re- ported P3 at L2. Figure 2, $C$ and $D$, shows the mean perceptual deviations of the tapped locations from the actual stimulus point to the opposite-side point. The mean deviations of $\mathrm{P} 1$ were plotted near 0 in both ISIs, and neither was significantly greater than 0 using the 50 or $80 \mathrm{~ms}$ ISI ( $p=0.25$ and 0.21 , respectively, uncorrected paired $t$ test). The mean deviation of $\mathrm{P} 2$ was significantly greater than that of $\mathrm{P} 1$ and $\mathrm{P} 3$ using a $50 \mathrm{~ms}(p<0.001$ and $p=0.021$, respectively, paired $t$ tests with the Holm correction) and $80 \mathrm{~ms}$ ( $p=0.0036$ and $p=0.026$, respectively) ISI. Finally, the mean deviation of $\mathrm{P} 3$ was significantly greater than that of $\mathrm{P} 1$ using a $50 \mathrm{~ms}(p=0.021)$ and $80 \mathrm{~ms}(p=0.019)$ ISI. Although there was no difference between the ISIs with the correction of multiple comparisons, the mean deviation of P3 was greater for the $50 \mathrm{~ms}$ ISI than for the $80 \mathrm{~ms}$ ISI without the correction ( $p=$ $0.038)$.

In this study, the perceptual deviation of P2 was greater than that of P3. Similar asymmetric reports have been observed in previous studies (Geldard, 1982; Flach and Haggard, 2006), whereas a symmetric report has also been observed (Kilgard and Merzenich, 1995). The latter study added one more reference pulse (P4) at L2 after P3; this four-tap sequence could be a cause of the symmetric report.

Figure $3 B$ shows a typical report from the subjects during experiment 2. Similar to P1 (blue dot), P2 (green dot) was reported at the actual stimulus point, L1. In addition, P3 (orange dot) was also reported at the actual stimulus point, L2. Although 
A

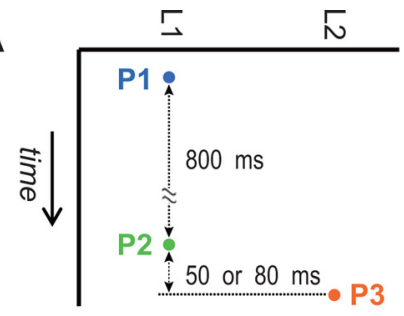

B
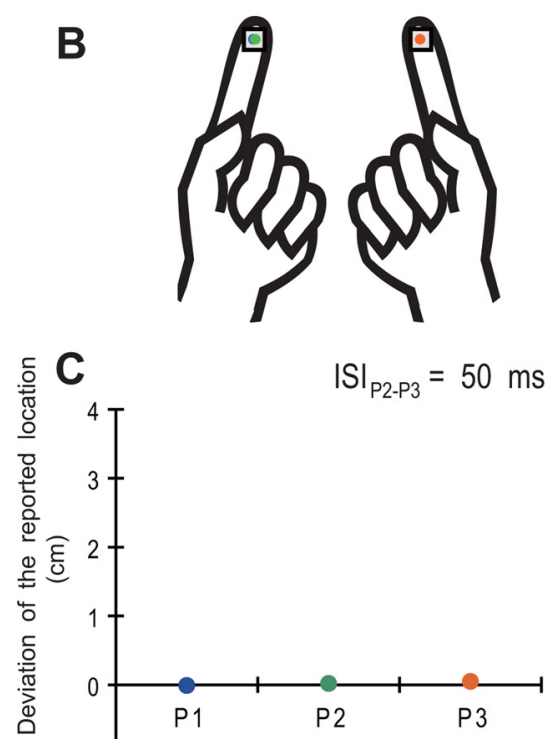

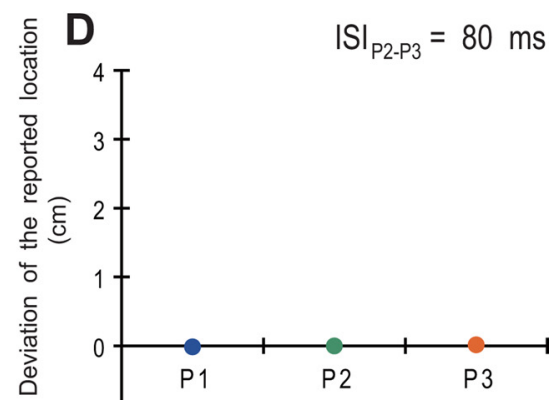

Figure 3. Stimulation without a stick (experiment 2). $\boldsymbol{A}$, The pulse sequence in this experiment was identical to that used in experiment 1 (see Fig. $2 A$ ). $\boldsymbol{B}$, Subjects received the pulse sequence via small plates, instead of a stick, held on each of the ventral pads of the index fingers. $\boldsymbol{C}, \boldsymbol{D}$, Mean perceptual deviations of the tapped locations from the actual stimulus point to the oppositeside point.

five of the eight subjects rarely reported small sensory saltation (1-2 $\mathrm{mm}$ ) of the tapped point of P2 [appearance rate (mean \pm SEM): $5.9 \pm 2.7 \%$ when ISI was $50 \mathrm{~ms}, 4.8 \pm 2.4 \%$ when ISI was $80 \mathrm{~ms}]$, there were no reports of tapped sensations occurring at any location outside the finger pads. Figure 3, $C$ and $D$, shows the mean perceptual deviation of the tapped location. All data are plotted at 0 , and there was no difference among or between the ISIs for P1, P2, or P3 ( $p \geq 0.18$, paired $t$ tests with the Holm correction). Thus, we did not reliably observe the cutaneous rabbit effect and never observed the illusory sensation outside the fingers under this condition. Connecting the fingers via a stick was therefore crucial for generating the illusory taps in the space between the fingers that were actually stimulated.

Comparisons across the experiments further supported the above results. In the comparison of $\mathrm{P} 1 \mathrm{~s}$, there was no difference between the experiments using a $50 \mathrm{~ms}(p=0.23$, uncorrected Welch's $t$ test) or $80 \mathrm{~ms}$ ( $p=0.37$ ) ISI. The perceptual deviation of P2, however, was significantly greater in experiment 1 (with stick) than in experiment 2 (without stick) using a $50 \mathrm{~ms}(p<$ 0.001 , Welch's $t$ test with the Holms correction) or $80 \mathrm{~ms}$ ( $p=$ $0.002)$ ISI. As for P3, the perceptual deviation was greater in experiment 1 than in experiment 2 using a $50 \mathrm{~ms}(p=0.0077)$ or $80 \mathrm{~ms}(p=0.016)$ ISI.

\section{Discussion}

To date, the cutaneous rabbit illusion has been observed only on the body (Geldard and Sherrick, 1972, 1983; Geldard, 1982; Kilgard and Merzenich, 1995; Eimer et al., 2005; Blankenburg et al., 2006; Flach and Haggard, 2006), and it is currently accepted that the illusion is associated with early unimodal somatotopic activity in S1 (Geldard, 1982; Geldard and Sherrick, 1983; Blankenburg et al., 2006; Flach and Haggard, 2006). Thus, in both physical and neuronal contexts, the cutaneous rabbit illusion has been confined to the body since it was first reported (Geldard and Sherrick, 1972). Our results, however, show that the illusory rabbit "hopped out of the body" and onto a stick held by subjects' index fingers. Our finding suggests that the effect involves not only the currently accepted somatotopic representation but also the representation of an external object that interacts with the body.

Over the past 10 years, psychophysical studies have continuously revealed flexible adaptability or scalability of human body perception (Ramachandran et al., 1995; Botvinick and Cohen, 1998; Yamamoto and Kitazawa, 2001b; Maravita et al., 2002; Yamamoto et al., 2005; Ehrsson, 2007), which has expanded the definition of a "body." For example, when mechanical stimuli were delivered to the tips of drumsticks held in each hand, the crosshand deficit of temporal order judgment (Yamamoto and Kitazawa, 2001a; Shore et al., 2002) also occurred when the sticks were crossed without a change in hand position (Yamamoto and Kitazawa, 2001b; Yamamoto et al., 2005). A similar phenomenon was observed in a reactiontime task (Maravita et al., 2002). This phenomenon indicated that the brain referred somatosensory signals from the hands to the tips of the sticks; the results were interpreted as behavioral evidence for the theory that a tool held by the hand is incorporated into the "body schema" (Iriki et al., 1996; Maravita and Iriki, 2004). In other words, the body schema is extended to the tool.

Our novel finding regarding the cutaneous rabbit illusion strengthens psychophysical evidence for this theory: the cutaneous rabbit, originally a somatotopic illusory effect, extended to the sensation of a stick held by the index fingers. Thus, the somatosensory interpolation between the stimulus points was also referred to an external object that interacts with the body. Accordingly, the cutaneous rabbit effect involves not only the intrinsic body-map representation but also a representation of the extended body schema resulting from body-object interactions.

It should also be noted that, in our results, the illusory tapping sensations occurred at locations where the stimuli were not actually delivered. In the preceding reports showing the referral of somatosensory sensation to external objects, the "tip" (i.e., the actual stimulus point) of the object was emphasized as the destination of the referral (Yamamoto and Kitazawa, 2001b; Yamamoto et al., 2005). It was furthermore argued that the tactile sensation was referred from the hand directly to the tips of the sticks without taking into account their intermediate routes. This conclusion was based on the observation that temporal order judgment was affected only by the position of the hands and the tips of the sticks and not by the shape of the sticks (Yamamoto et 
al., 2005). Our findings, however, showed that the illusory tapping sensation occurs at intermediate locations between the stimulus points on a stick. Thus, the present observation clearly indicates that the brain not only took into account the action points of stimuli on the stick but also considered the intermediate structure between the points. This suggests that, according to the task demand, the brain can incorporate the whole structure of an object into the body scheme. We speculate that this perceptual faculty contributes to a computation for predicting the dynamics of an object when the subject manipulates the object (Ahmed et al., 2008).

The neurophysiological mechanisms of this phenomenon are currently unclear; however, our data indicate that the neurophysiological knowledge regarding the cutaneous rabbit illusion or the representation of objects/tools interacting with the body needs to be updated. For example, previous neurophysiological studies on monkeys proposed that the intraparietal cortex was a neural substrate in which the representation of the body schema is extended by tool handling (Iriki et al., 1996, 2001). In fMRI studies on humans, the neural correlates for the perception of body-object interactions were observed in the posterior parietal regions (Naito and Ehrsson, 2006; Naito et al., 2008). Our findings, therefore, may suggest that the cutaneous rabbit effect can also involve the posterior parietal area in the context of holding an external object/tool.

In addition to $S 1$, the neural correlates for the cutaneous rabbit illusion were also observed in the premotor and prefrontal cortices, and these areas have been assumed to be involved in the top-down modulation of the somatosensory integrative processing in S1 (Blankenburg et al., 2006). The premotor area constructs functional connections with the posterior parietal regions in relation to the body-space representation (Rizzolatti et al., 2002). The premotor-intraparietal circuit was reported as a neural correlate of the rubber hand illusion (Ehrsson et al., 2004). This illusion indicated that cleverly devised visual stimuli could induce a referral of tactile signals to mimic the body part (Botvinick and Cohen, 1998). Moreover, these brain areas were involved in the somatic rubber hand illusion that was induced only by tactile stimuli (Ehrsson et al., 2005). The premotor-intraparietal circuit seems to be a plausible, essential neural substrate for generating the illusory rabbit on the stick.

We cannot exclude the possibility of an essential involvement of $\mathrm{S} 1$ in the illusory rabbit on the stick. A recent fMRI study showed that the activity of the primary sensorimotor area, including S1 (BA 1), increased in the bimanual manipulation of an object (Theorin and Johansson, 2007). In the task, interactions between the hands should be transmitted via the object. The brain thus should take account of the property or dynamics of the object to perform the task. Taking the S1-rabbit association and our findings into consideration, the observation by Theorin and Johansson might reflect some transient plasticity in S1 according to the object handling. Although these hypotheses are merely speculative, the psychophysical phenomenon presented herein provides a novel and effective clue for investigating how the human brain coordinates the body schema according to add-on contexts, such as body-object interactions.

\section{References}

Ahmed AA, Wolpert DM, Flanagan JR (2008) Flexible representations of dynamics are used in object manipulation. Curr Biol 18:763-768.

Blankenburg F, Ruff CC, Deichmann R, Rees G, Driver J (2006) The cutaneous rabbit illusion affects human primary sensory cortex somatotopically. PLoS Biol 4:e69.
Botvinick M, Cohen J (1998) Rubber hands "feel" touch that eyes see. Nature 391:756.

Dennett DC (1991) Consciousness explained. Boston: Little, Brown.

Eagleman DM, Sejnowski TJ (2000) Motion integration and postdiction in visual awareness. Science 287:2036-2038.

Ehrsson HH (2007) The experimental induction of out-of-body experiences. Science 317:1048.

Ehrsson HH, Spence C, Passingham RE (2004) That's my hand! Activity in premotor cortex reflects feeling of ownership of a limb. Science 305:875-877.

Ehrsson HH, Holmes NP, Passingham RE (2005) Touching a rubber hand: feeling of body ownership is associated with activity in multisensory brain areas. J Neurosci 25:10564-10573.

Eimer M, Forster B, Vibell J (2005) Cutaneous saltation within and across arms: a new measure of the saltation illusion in somatosensation. Percept Psychophys 67:458-468.

Flach R, Haggard P (2006) The cutaneous rabbit revisited. J Exp Psychol Hum Percept Perform 32:717-732.

Geldard FA (1982) Saltation in somesthesis. Psychol Bull 92:136-175.

Geldard FA, Sherrick CE (1972) The cutaneous "rabbit": a perceptual illusion. Science 178:178-179.

Geldard FA, Sherrick CE (1983) The cutaneous saltatory area and presumed neural basis. Percept Psychophys 33:299-304.

Goldreich D (2007) A Bayesian perceptual model replicates the cutaneous rabbit and other tactile spatiotemporal illusions. PLoS ONE 2:e333.

Helson H (1930) The tau effect-an example of psychological relativity. Science 71:536-537.

Iriki A, Tanaka M, Iwamura Y (1996) Coding of modified body schema during tool use by macaque postcentral neurones. Neuroreport 7:23252330.

Iriki A, Tanaka M, Obayashi S, Iwamura Y (2001) Self-images in the video monitor coded by monkey intraparietal neurons. Neurosci Res 40:163-173.

Jones FN (1956) Space-time relationships in somesthetic localization. Science 124:484.

Kilgard MP, Merzenich MM (1995) Anticipated stimuli across skin. Nature 373:663.

Maravita A, Iriki A (2004) Tools for the body (schema). Trends Cogn Sci 8:79-86.

Maravita A, Spence C, Kennett S, Driver J (2002) Tool-use changes multimodal spatial interactions between vision and touch in normal humans. Cognition 83:B25-B34.

Miyazaki M, Yamamoto S, Uchida S, Kitazawa S (2006) Bayesian calibration of simultaneity in tactile temporal order judgment. Nat Neurosci 9:875-877.

Mountcastle VB (1984) Central nervous mechanisms in mechanoreceptive sensibility. In: Handbook of physiology (Darian-Smith, ed), pp 789-878. Bethesda, MD: American Physiological Society.

Naito E, Ehrsson HH (2006) Somatic sensation of hand-object interactive movement is associated with activity in the left inferior parietal cortex. J Neurosci 26:3783-3790.

Naito E, Scheperjans F, Eickhoff SB, Amunts K, Roland PE, Zilles K, Ehrsson HH (2008) Human superior parietal lobule is involved in somatic perception of bimanual interaction with an external object. J Neurophysiol 99:695-703.

Penfield W, Boldrey E (1937) Somatic motor and sensory representation in cerebral cortex of man as studied by electrical stimulation. Brain 60:389-443.

Ramachandran VS, Rogers-Ramachandran D, Cobb S (1995) Touching the phantom limb. Nature 377:489-490.

Rizzolatti G, Fogassi L, Gallese V (2002) Motor and cognitive functions of the ventral premotor cortex. Curr Opin Neurobiol 12:149-154.

Shore DI, Spry E, Spence C (2002) Confusing the mind by crossing the hands. Brain Res Cogn Brain Res 14:153-163.

Theorin A, Johansson RS (2007) Zones of bimanual and unimanual preference within human primary sensorimotor cortex during object manipulation. Neuroimage 36:T2-T15.

Yamamoto S, Kitazawa S (2001a) Reversal of subjective temporal order due to arm crossing. Nat Neurosci 4:759-765.

Yamamoto S, Kitazawa S (2001b) Sensation at the tips of invisible tools. Nat Neurosci 4:979-980.

Yamamoto S, Moizumi S, Kitazawa S (2005) Referral of tactile sensation to the tips of L-shaped sticks. J Neurophysiol 93:2856-2863. 